ERRATUM

doi:10.1038/nature11453

\title{
Erratum: Integrated genomic analyses of ovarian carcinoma
}

The Cancer Genome Atlas Research Network

Nature 474, 609-615 (2011); doi:10.1038/nature10166

In this Letter, J. G. Vockley was incorrectly listed as J. B. Vockley. Also, several authors were inadvertently omitted from the genome sequencing centre group: C. J. Creighton, from the Human Genome Sequencing Center at the Baylor College of Medicine; R. Nichol and S. Fisher, from the Sequencing Platform at the Broad Institute; and E. Upfal, from the Department of Computer Science at Brown University. In addition, J. Samayoa, from the Institute for Computational Medicine, was inadvertently missing from the genome data analysis centre group at the Johns Hopkins University. These have been corrected in the HTML and PDF versions of the manuscript. 